\title{
Glucocorticoid enhancement of memory requires arousal-induced noradrenergic activation in the basolateral amygdala
}

\author{
Benno Roozendaal* , Shoki Okuda*‡, Eddy A. Van der Zee`, and James L. McGaugh* ${ }^{\star \dagger}$ \\ *Center for the Neurobiology of Learning and Memory, and Department of Neurobiology and Behavior, University of California, Irvine, CA 92697-3800; \\ ${ }^{\ddagger}$ CNS Disorder Research, Tsukuba Research Institute, Banyu Pharmaceutical Co., Ltd., Tsukuba, Ibaraki 300-2611, Japan; and §Department of Molecular \\ Neurobiology, University of Groningen, P.O. Box 14, 9750 AA, Haren, The Netherlands
}

Contributed by James L. McGaugh, March 8, 2006

\begin{abstract}
Considerable evidence indicates that glucocorticoid hormones enhance the consolidation of long-term memories for emotionally arousing experiences but not that for less arousing or neutral information. However, previous studies have not determined the basis of such arousal-induced selectivity. Here we report the finding that endogenous noradrenergic activation of the basolateral complex of the amygdala (BLA) induced by emotional arousal is essential in enabling glucocorticoid memory enhancement. Corticosterone administered immediately after object recognition training enhanced 24-h memory of naïve male rats but not that of rats previously habituated to the training context in order to reduce novelty-induced emotional arousal. The $\beta$-adrenoceptor antagonist propranolol administered either systemically or into the BLA blocked the corticosterone-induced memory enhancement. Further, in habituated rats, corticosterone activated BLA neurons, as assessed by phosphorylated CAMP response element binding (pCREB) immunoreactivity levels, and enhanced memory only when norepinephrine release was stimulated by administration of the $\alpha_{2}$-adrenoceptor antagonist yohimbine. These findings strongly suggest that synergistic actions of glucocorticoids and emotional arousal-induced noradrenergic activation of the BLA constitute a neural mechanism by which glucocorticoids may selectively enhance memory consolidation for emotionally arousing experiences.
\end{abstract}

corticosterone | emotional arousal | norepinephrine | object recognition memory consolidation

$\mathbf{E}_{\mathrm{r}}^{\mathrm{x}}$ xtensive evidence indicates that emotionally arousing experiences are typically well remembered $(1,2)$. Investigations of the underlying neurobiological mechanisms suggest that glucocorticoids, released from the adrenal cortex by emotional arousal, play a key role in the strengthening of new memory traces (3-5). Recent findings of both animal and human studies suggest that glucocorticoids may selectively modulate the consolidation of memories of emotionally arousing stimuli (6-9) or those of experiences occurring during states of emotional arousal (10). Such findings imply that glucocorticoids interact with some other component of emotional arousal in influencing memory consolidation. We reported evidence that selective blockade of noradrenergic activity in the basolateral complex of the amygdala (BLA) of rats with infusions of a $\beta$-adrenoceptor antagonist prevented the memory-enhancing effects of glucocorticoids administered either systemically or into selected brain regions (11-13). Such findings, considered along with the evidence that emotional arousal activates the BLA (14-19) and induces the release of norepinephrine within the $\operatorname{BLA}(20,21)$, suggest that emotional arousal-induced noradrenergic activation within the BLA may be essential in enabling glucocorticoid effects on memory consolidation. However, because prior experiments investigating glucocorticoid-noradrenergic interactions on memory consolidation have used training conditions that induce the release of high levels of norepinephrine in the $\operatorname{BLA}(20,21)$, such studies did not determine whether glucocorticoid enhancement of memory requires emotional arousal-induced noradrenergic activation of the BLA or, alternatively, whether glucocorticoids enhance memory consolidation by activating the release of norepinephrine in the BLA.

The aim of the present experiments was to investigate whether noradrenergic activation of the BLA triggered specifically by the emotionally arousing properties of the training experience is critically involved in enabling glucocorticoid effects on memory consolidation. In these experiments, rats were trained on an object recognition task. Although no rewarding or aversive stimulation is used during object recognition training (22), we previously had found that such training stimulates novelty-induced emotional arousal in naïve rats and that extensive prior habituation to the experimental context attenuates such arousal during training (9). Thus, rats were exposed to the objects while in a state of arousal or in a less aroused state. We previously found that corticosterone selectively enhances the consolidation of object recognition memory when administered to nonhabituated (i.e., emotionally aroused) rats immediately after the training trial (9). Thus, if noradrenergic activation of the BLA induced by the novelty stress is necessary for enabling the glucocorticoid effect on memory consolidation, then (i) blockade of noradrenergic activation should prevent corticosterone-induced memory enhancement in nonhabituated rats, (ii) pharmacological stimulation of norepinephrine release should enable corticosterone-induced memory enhancement for objects experienced in a familiar low-arousing context, and (iii) $\beta$-adrenoceptor activation within the BLA should be critically involved in enabling these glucocorticoid effects on memory consolidation. Our findings provide strong support for each of these implications. Additionally, we found that corticosterone activated BLA neurons only when noradrenergic activity was stimulated concurrently.

\section{Results}

Blockade of Noradrenergic Activity Prevents Corticosterone Effects on Memory Enhancement in Nonhabituated Rats. Context-naïve male Sprague-Dawley rats were trained on an object recognition task and received a vehicle solution or corticosterone $(0.3,1.0$, or 3.0 $\mathrm{mg} / \mathrm{kg}$, s.c.) either alone or coadministered with the $\beta$-adrenoceptor antagonist propranolol $(3.0 \mathrm{mg} / \mathrm{kg}$, s.c.) immediately after the training. During the 3-min training trial, before drug treatment, the groups spent comparable times exploring each of two identical objects (one-sample $t$ tests: $P>0.33$ ) and also did not differ in total times exploring both objects (one-way ANOVA: $F_{7,77}=1.62 ; P=0.14$; see Table 2, which is published as supporting information on the PNAS web site). We previously

Conflict of interest statement: No conflicts declared.

Abbreviations: BLA, basolateral complex of the amygdala; PCREB, phosphorylated CAMP response element binding; $\mathrm{PTH}$, phosphorylated tyrosine hydroxylase.

tTo whom correspondence may be addressed. E-mail: broozend@uci.edu or jlmcgaug@uci.edu.

() 2006 by The National Academy of Sciences of the USA 

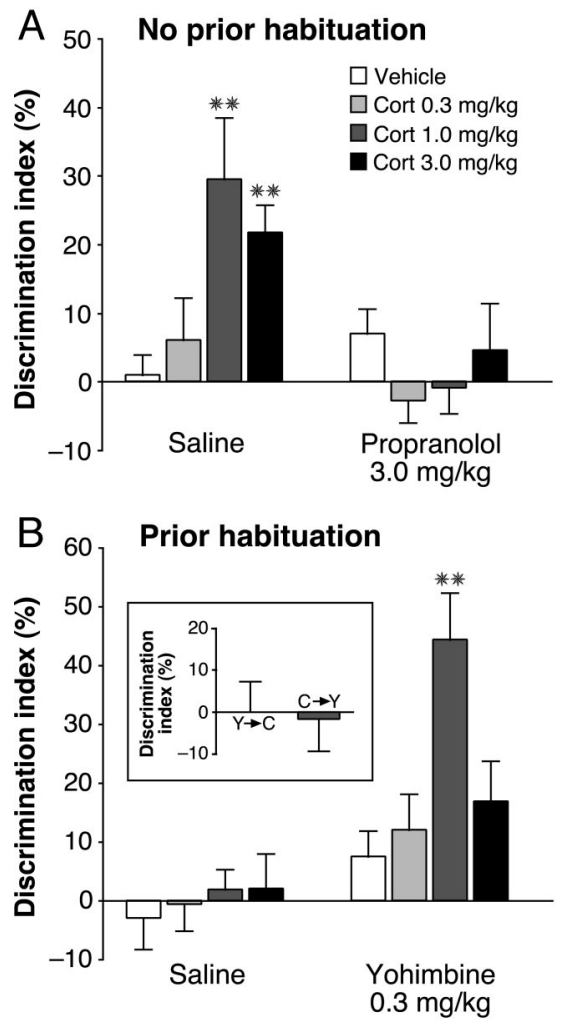

Fig. 1. Glucocorticoid effects on memory consolidation for object recognition training require noradrenergic activation. Data represent discrimination index (\%) on a 24-h retention trial, expressed as mean \pm SEM. (A) Effects of immediate posttraining administration of the $\beta$-adrenoceptor antagonist propranolol $(3.0 \mathrm{mg} / \mathrm{kg}$, s.c.) on corticosterone-induced enhancement of object recognition memory in naïve rats. $* *, P<0.0001$ vs. vehicle $(n=8-12$ per group). (B) Effect of coadministration of the $\alpha_{2}$-adrenoceptor antagonist yohimbine $(0.3 \mathrm{mg} / \mathrm{kg}$, s.c.) with corticosterone on object recognition memory in habituated rats. **, $P<0.0001$ vs. vehicle ( $n=9-17$ per group). ( $B$ Inset) Effect of posttraining injections of yohimbine $(0.3 \mathrm{mg} / \mathrm{kg}$, s.c.) and corticosterone $(1.0 \mathrm{mg} / \mathrm{kg}$, s.c.) separated by a $4-\mathrm{h}$ delay. $Y \rightarrow C$, yohimbine administered immediately after training and corticosterone $4 \mathrm{~h}$ later; $\mathrm{C} \rightarrow \mathrm{Y}$, corticosterone administered immediately after training and yohimbine $4 \mathrm{~h}$ later.

found that rats readily remember such training when tested at a 1-h retention interval (9). Two-way ANOVA for 24-h retention performance revealed significant corticosterone $\left(F_{3,77}=2.70\right.$; $P<0.05)$ and propranolol effects $\left(F_{1,77}=10.52 ; P<0.05\right)$, as well as a significant interaction between both factors $\left(F_{3,77}=\right.$ 4.02; $P<0.05)$. On the 24 -h retention test, the vehicle-treated group did not show significant preference for the novel object versus the object explored during training (one-sample $t$ test: $P=$ 0.76 ), but rats given the 1.0 and $3.0 \mathrm{mg} / \mathrm{kg}$ doses of corticosterone posttraining exhibited a highly significant preference for the novel object (one-sample $t$ tests: $P<0.0004$; Fig. 1A). Blockade of noradrenergic activity with posttraining injections of propranolol administered alone did not affect object recognition retention performance $(P=0.43$ vs. vehicle) but prevented the retention enhancement induced by corticosterone $(P>0.24$ vs. propranolol). The groups did not differ in total times exploring both objects during the retention test trial (one-way ANOVA: $F_{7,77}=1.26 ; P=0.28$; see Table 2 ). These findings indicate that noradrenergic activity is essential to enable glucocorticoid effects on memory consolidation with training conditions that produce mild novelty-induced emotional arousal and thus not only, as reported previously, with highly arousing training (11-13, 23).
Table 1. Effect of yohimbine on object recognition memory

\begin{tabular}{lcc} 
Yohimbine & No prior habituation & Prior habituation \\
\hline Saline & $-1.8 \pm 6.5$ & $2.0 \pm 3.3$ \\
$0.1 \mathrm{mg} / \mathrm{kg}$ & $15.8 \pm 4.2^{*}$ & $1.5 \pm 3.6$ \\
$0.3 \mathrm{mg} / \mathrm{kg}$ & $35.8 \pm 4.8^{*}$ & $-2.2 \pm 7.3$ \\
$1.0 \mathrm{mg} / \mathrm{kg}$ & $16.5 \pm 5.3^{*}$ & $-2.2 \pm 4.0$ \\
$3.0 \mathrm{mg} / \mathrm{kg}$ & $-0.01 \pm 5.4$ & $27.8 \pm 4.0^{*}$
\end{tabular}

Data represent discrimination index (\%) on the 24-h retention test trial, expressed as mean \pm SEM. One-way ANOVA revealed significant treatment effects in naïve rats $\left(F_{4.52}=8.88, P<0.0001\right)$ as well as in habituated rats $\left(F_{4,55}=9.77, P<0.0001\right)$. Yohimbine was administered subcutaneously immediately after the training trial. *, $P<0.05$ as compared with the corresponding saline group ( $n=10-17$ per group).

Pharmacologically Induced Noradrenergic Activation Mimics the Effect of Emotional Arousal in Enabling Corticosterone Effects on Memory Enhancement in Habituated Rats. As indicated, we previously reported that corticosterone does not enhance the consolidation of object recognition memory in rats given extensive prior habituation to the training context to reduce noveltyinduced emotional arousal (9). If the failure of corticosterone to enhance memory consolidation in context-habituated rats is due selectively to insufficient arousal-induced noradrenergic activation, then posttraining pharmacological augmentation of noradrenergic activity should provide the activation normally produced by novelty stress and enable glucocorticoid enhancement of memory consolidation. To examine this implication, a low dose of the $\alpha_{2}$-adrenoceptor antagonist yohimbine $(0.3 \mathrm{mg} / \mathrm{kg}$, s.c.), which increases norepinephrine levels in the brain (24), was administered to habituated rats either alone or together with corticosterone, immediately after object recognition training (Fig. 1B). This dose of yohimbine was selected because it does not enhance the consolidation of object recognition memory in habituated rats (Table 1). During the 3-min training trial, before drug treatment, the groups spent comparable times exploring each of two identical objects (one-sample $t$ tests: $P>0.39$ ) and also did not differ in total times exploring both objects (one-way ANOVA: $F_{7,80}=0.45 ; P=0.79$; see Table 2 ). Two-way ANOVA for 24-h retention performance revealed significant corticosterone $\left(F_{3,89}=5.84 ; P<0.005\right)$ and yohimbine effects $\left(F_{1,89}=\right.$ $24.88 ; P<0.0001)$, as well as a significant interaction between both factors $\left(F_{3,89}=3.97 ; P<0.01\right)$. Posttraining administration of corticosterone alone failed to induce a preference for the novel object on the retention test (one-sample $t$ tests: $P>0.62$ ). Moreover, although yohimbine administered alone also did not affect retention performance $(P=0.18$ vs. vehicle $)$, corticosterone administered concurrently with yohimbine induced dosedependent retention enhancement $(1.0 \mathrm{mg} / \mathrm{kg}: P<0.0001$ vs. yohimbine) comparable with that of rats not given prior habituation. Posttraining injections of the two drugs separated by a 4-h delay did not induce a preference for the novel object on the retention trial (one-sample $t$ tests: $P>0.83$; Fig. $1 B$ Inset). These findings thus indicate that glucocorticoid effects on memory consolidation normally require emotional arousal-induced noradrenergic activation. However, pharmacologically stimulated noradrenergic activity fully mimics the effects of emotional arousal in enabling glucocorticoid enhancement of memory consolidation under low-arousing training conditions.

Corticosterone Effects on Memory Enhancement Require Noradrenergic Activity Within the Basolateral Amygdala. Findings of animal and human studies provide extensive evidence that emotionally arousing experiences activate the amygdala and that such activity (especially of the BLA) modulates memory consolidation $(5$, 14-19). Furthermore, emotionally arousing training experiences induce the release of norepinephrine within the amygdala $(20$, 
A Basolateral amygdala

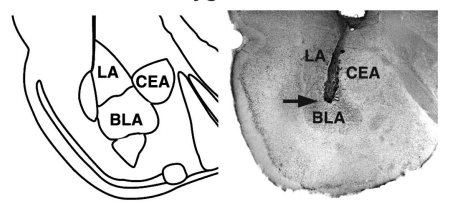

B Hippocampus
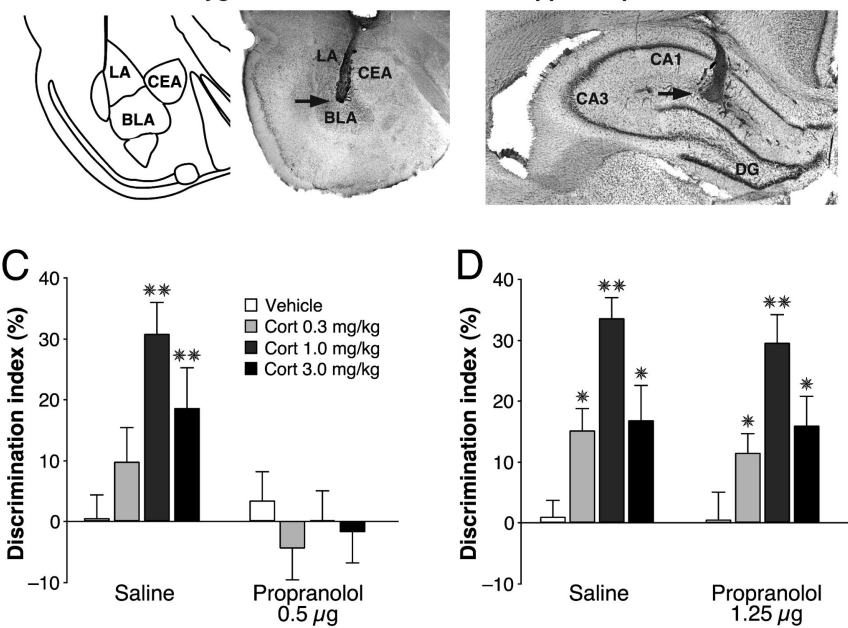

Fig. 2. Activation of $\beta$-adrenoceptors in the BLA, but not the hippocampus, is required for enabling corticosterone effects on object recognition memory. Data represent discrimination index (\%) on a 24-h retention trial, expressed as mean \pm SEM. ( $A$ and $B$ ) Diagram and photomicrograph illustrating placement of infusion needle tip in the BLA and hippocampus. The arrow points to the needle tip. (C) Effect of posttraining administration of the $\beta$-adrenoceptor antagonist propranolol (0.5 $\mu \mathrm{g}$ per hemisphere) into the BLA on corticosterone-induced enhancement of object recognition memory in nonhabituated rats. **, $P<0.0001$ vs. vehicle ( $n=8-11$ per group). (D) Effect of posttraining administration of the $\beta$-adrenoceptor antagonist propranolol $(1.25 \mu \mathrm{g}$ per hemisphere) into the hippocampus on corticosterone-induced enhancement of object recognition memory in nonhabituated rats. *, $P<0.05 ; * *, P<$ 0.0001 vs. vehicle ( $n=10-14$ per group).

21). To examine whether glucocorticoid effects on object recognition memory depend on noradrenergic activity within the BLA, propranolol ( $0.5 \mu \mathrm{g}$ per hemisphere; see Fig. $2 A$ for infusion site) was infused bilaterally into the BLA of nonhabituated rats immediately after object recognition training, and corticosterone was then immediately administered systemically. Because the hippocampus is also highly sensitive to the effects of glucocorticoids $(25,26)$ but does not seem to play a critical role in object recognition memory $(27,28)$, other groups of rats received posttraining infusions of propranolol $(1.25 \mu \mathrm{g}$ per hemisphere) into the dorsal hippocampus (see Fig. $2 B$ for infusion site). The groups did not differ in total exploration times of the two objects during training (one-way ANOVA: BLA, $F_{7,71}=1.08, P=0.39$; hippocampus, $\left.F_{7,86}=0.41, P=0.89\right)$ or testing (BLA: $F_{7,71}=0.69, P=0.68$; hippocampus: $F_{7,86}=0.20$, $P=0.98$; see Table 3 , which is published as supporting information on the PNAS web site). Two-way ANOVA for 24-h retention performance of rats administered vehicle or corticosterone systemically and either saline or propranolol into the BLA revealed significant corticosterone $\left(F_{3,71}=2.95 ; P<0.05\right)$ and propranolol effects $\left(F_{1,71}=17.66 ; P<0.0001\right)$, as well as a significant interaction between both factors $\left(F_{3,71}=3.49 ; P<\right.$ 0.05). Post hoc analysis revealed that posttraining intra-BLA infusions of propranolol blocked 24-h retention enhancement induced by immediate posttraining administration of corticosterone $(P>0.31$ vs. propranolol; Fig. $2 C)$. In contrast, posttraining infusions of propranolol into the dorsal hippocampus did not block the corticosterone-induced enhancement (Fig. 2D). These findings thus indicate that glucocorticoid-induced enhancement of memory consolidation depends selectively on noradrenergic activation within the BLA.

Corticosterone Effects on Basolateral Amygdala Neuronal Activity Require Arousal-Induced Noradrenergic Activation. To examine whether arousal induced by object recognition training induces noradrenergic activation in the BLA and whether such activation is necessary for mediating glucocorticoid effects on BLA activity, we first determined the immunoreactivity for phosphorylated (i.e., activated) tyrosine hydroxylase (pTH) (29), the rate-limiting enzyme in the biosynthesis of norepinephrine (30), in brains of habituated and nonhabituated rats. One-way ANOVA for pTH immunoreactivity in the BLA revealed a significant group effect $\left(F_{7,34}=9.81 ; P<0.0001\right)$. Object recognition training increased the density of pTH-positive fibers in the BLA of nonhabituated rats by $\approx 100 \% 3 \mathrm{~h}$ after training $(P<0.0001$ vs. home cage controls; Fig. $3 A$ and $B)$. Furthermore, double staining with dopamine- $\beta$-hydroxylase, the enzyme that converts dopamine into norepinephrine, revealed a nearly $100 \%$ overlap with pTH immunoreactivity (data not shown), indicating that arousal induced by object recognition training induces a marked increase in $\mathrm{TH}$ activity and local synthesis of norepinephrine in the BLA. Prior habituation of rats to the experimental context significantly reduced the density of pTH-positive fibers assessed $3 \mathrm{~h}$ after object recognition training $(P<0.001$ vs. nonhabituated counterparts $)$ and induced only a trend toward an increase from home cage controls that had received similar habituation training $(\approx 28 \%$ increase; $P=0.06)$. Training-induced increases in pTH immunoreactivity in the BLA resulted from phosphorylation of existing $\mathrm{TH}$, because habituated and nonhabituated rats did not differ in total (i.e., phosphorylated and nonphosphorylated) $\mathrm{TH}$ immunoreactivity in BLA fibers and nerve terminals $(P=0.54$; data not shown). Importantly, corticosterone $(1.0 \mathrm{mg} / \mathrm{kg})$ administered posttraining did not alter pTH immunoreactivity in the BLA of either habituated or nonhabituated rats $(P>0.62$; Fig. $3 A$ and $B)$, indicating that, under these conditions, corticosterone did not enhance memory consolidation by increasing the local synthesis of norepinephrine in the BLA. Furthermore, arousal-induced increases in noradrenergic activity, as assessed by pTH, were found in the BLA but not the hippocampus. Prior habituation, object recognition training, or corticosterone administration all failed to alter pTH immunoreactivity levels in the dentate gyrus (one-way ANOVA: $F_{7,34}=0.24, P=0.97$; Fig. $3 C$ ) or CA1 region of the hippocampus (data not shown).

Our finding that corticosterone-induced memory enhancement requires noradrenergic activation but does not increase pTH immunoreactivity in BLA fibers and nerve terminals suggests that corticosterone interacts with training-induced noradrenergic activation at a postsynaptic level, possibly via an activation of membrane-bound glucocorticoid receptors (31), in increasing BLA neuronal activity (13). To assess postsynaptic BLA neuronal activity, we determined immunoreactivity for the phosphorylated form of the transcription factor cAMP response element-binding ( $\mathrm{pCREB}$ ) protein. Noradrenergic stimulation induces pCREB activation $(32,33)$, and several findings have implicated CREB phosphorylation in the amygdala in the modulation of memory consolidation (33-35). One-way ANOVA for pCREB immunoreactivity in the BLA revealed a significant group effect $\left(F_{7,34}=5.58 ; P<0.0005\right)$. In nonhabituated rats, posttraining corticosterone $(1.0 \mathrm{mg} / \mathrm{kg})$ administration significantly increased the number of pCREB-positive neurons in the BLA 3 h after training $(P<0.05$ vs. vehicle; Fig. $3 A$ and $B)$. However, in habituated rats, posttraining corticosterone or yohimbine administered alone did not significantly increase the number of pCREB-positive BLA neurons. Importantly, however, corticosterone administered together with yohimbine significantly increased pCREB immunoreactivity in the BLA $(P<$ 0.01 vs. yohimbine). Thus, these findings indicate that corticosterone activates the BLA, as assessed by pCREB, only under training conditions that induce sufficient noradrenergic activation within the BLA. In contrast, no training-related or treatment-related increases in pCREB immunoreactivity were found in either the dentate gyrus (one-way ANOVA: $F_{7,34}=0.19, P=$ 
A

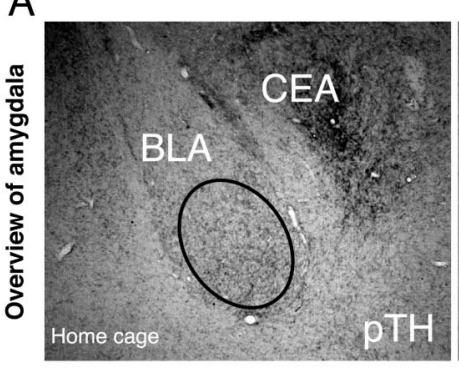

No prior habituation

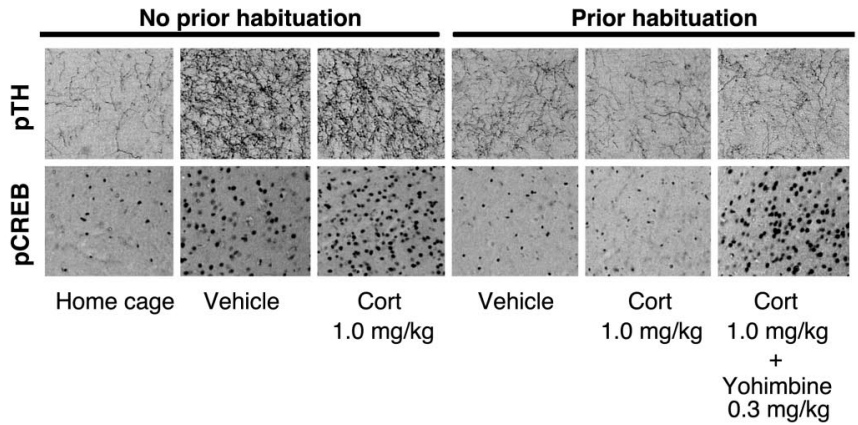

B
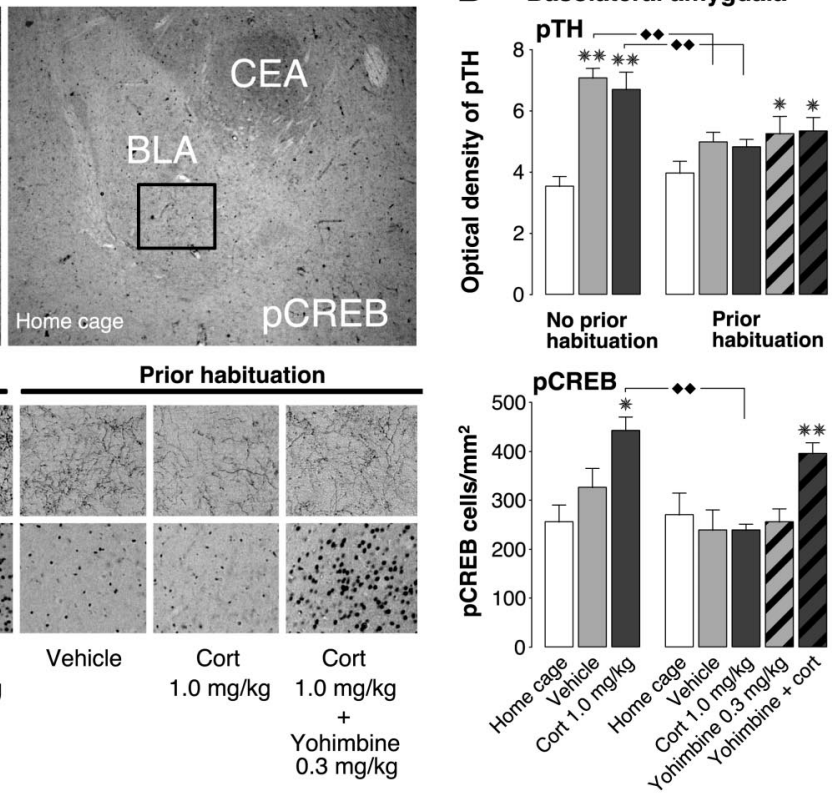

C Dentate gyrus

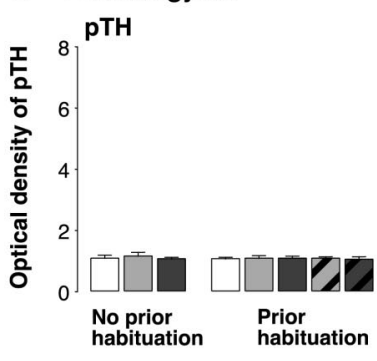

pCREB

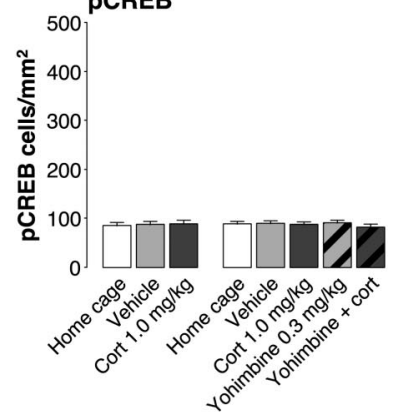

Fig. 3. Prior habituation attenuates the effects of object recognition training and corticosterone (Cort) injection on noradrenergic activity within the BLA. ( $A$ )

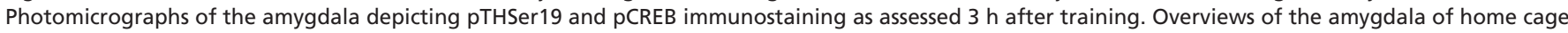

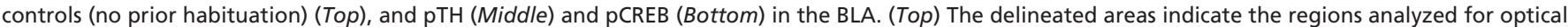
density (pTH) or cell counts (pCREB). ( $B$ and $C$ ) Summary of the immunocytochemistry data for the BLA and dentate gyrus (mean \pm SEM). *, $P<0.05 ; * *, P<0.01$

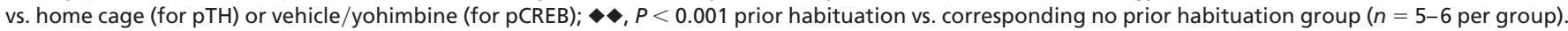
CEA, central amygdala.

0.98; Fig. $3 C)$ or CA1 region of the hippocampus $\left(F_{7,34}=0.04\right.$, $P=1.00$; data not shown).

\section{Discussion}

The objective of this study was to investigate whether glucocorticoid effects on memory consolidation require emotional arousalinduced increases in noradrenergic activity. To examine this issue, the present experiments used the strategy of training rats on an object recognition task in an experimental context that induces either an aroused or less aroused emotional state. In an earlier study, we found that extensive prior habituation to the training context decreases emotional arousal induced by the training, as assessed by both behavioral and neuroendocrine measures (9). Corticosterone administered immediately after the training experience selectively enhanced memory of rats that were naïve to the experimental context. Results of human studies examining the role of emotional arousal by varying the emotionally arousing content of the learning material have also suggested that glucocorticoids (administered before learning) selectively enhance memory for emotionally arousing information (6), although some studies reported different effects $(8,36)$. Consistent with our findings, Abercrombie et al. (10) reported that levels of endogenous cortisol correlated with enhanced memory consolidation only in individuals who were emotionally aroused. It is, of course, possible that the glucocorticoid-induced memory enhancement seen in these human studies was due, at least in part, to increased attentional bias to arousing stimuli experienced during the encoding. Importantly, in the present experiments, the drugs were administered immediately after training to influence memory consolidation and to exclude any influence of the drugs on information encoding. Moreover, total exploration of the objects exhibited during training was not greater in nonhabituated (i.e., emotionally aroused) rats (see Table 2) and retention performance of habituated and nonhabituated vehicle-treated controls did not differ from each other.
Blockade of noradrenergic activity with a $\beta$-adrenoceptor antagonist administered immediately after object recognition training to nonhabituated (i.e., emotionally aroused) rats prevented the corticosterone-induced memory enhancement. Conversely, stimulating noradrenergic activity with the $\alpha_{2}$ adrenoceptor antagonist yohimbine enabled glucocorticoid memory enhancement in rats that had a reduced emotional arousal response because of prior habituation. Thus, although emotional arousal is known to induce a myriad of peripheral and central physiological responses (37), our findings indicate that selective manipulation of noradrenergic activity after training is sufficient to either block or mimic the influence of emotional arousal in enabling the facilitating effects of posttraining glucocorticoid administration on memory consolidation. Moreover, the finding that posttraining administrations of yohimbine and corticosterone separated by a 4-h delay did not induce memory enhancement supports the hypothesis that both arousal systems need to be activated together. Thus, these findings strongly suggest that glucocorticoids enhance memory for emotionally arousing experiences because of critical interactions with endogenous noradrenergic activation induced by the training experience and make it very unlikely that glucocorticoids enhance memory by stimulating the release of norepinephrine or that basal levels of norepinephrine are sufficiently high to enable glucocorticoid effects on memory consolidation.

Our findings further indicate that glucocorticoid-induced enhancement of memory consolidation for object recognition training requires, in particular, noradrenergic activation within the BLA. We found that object recognition training in a novel, but not familiar, environment increased pTH immunoreactivity (i.e., noradrenergic activation) in the BLA, and that blockade of this noradrenergic activity with a $\beta$-adrenoceptor antagonist infused into the BLA prevented glucocorticoid enhancement of object recognition memory. Corticosterone administration did not alter pTH immunoreactivity in the BLA of either habituated 
or nonhabituated rats. However, corticosterone required noradrenergic activation induced either by the training experience or the yohimbine administration to increase BLA neuronal activity, as assessed by increases in pCREB immunoreactivity, a transcription factor necessary for memory processes $(34,38,39)$. These findings thus suggest that corticosterone interacts with training-induced noradrenergic activation at a postsynaptic level, possibly via an activation of membrane-bound glucocorticoid receptors (31), in increasing BLA neuronal activity and enhancing memory consolidation (13). Such findings fit with previous evidence on an inhibitory avoidance task indicating that glucocorticoids or the specific glucocorticoid receptor agonist RU 28362 enhance memory consolidation by potentiating $\beta$-adrenoceptor-cAMP-dependent protein kinase signaling in the BLA (13). Moreover, previous studies reported similar synergistic effects of glucocorticoids and the noradrenergic system on pCREB activation in regulating other cellular functions $(40,41)$. Such findings indicating that noradrenergic activation within the BLA plays a central role in regulating glucocorticoid effects on memory consolidation are also consistent with extensive prior evidence indicating that noradrenergic activity of the BLA, but not the adjacent central nucleus, is critically involved in memory consolidation and mediates the memory-modulatory effects of drugs affecting a variety of neurotransmitter systems $(5,42-44)$. Furthermore, the findings are in accord with evidence from functional imaging studies in human subjects indicating that amygdala activity during encoding of emotional activity correlates with subsequent retention performance (14) and that both the amygdala activity and subsequent retention are attenuated by propranolol administered before encoding $(16,18)$. In view of the extensive evidence that BLA activation influences memory consolidation via projections to other brain regions involved in processing recent information (5), it is likely that the enhanced memory seen in the present study was mediated by influencing activity in the perirhinal and insular cortices (45), because these brain regions are known to be critically involved in object recognition memory $(27,46-48)$.

In contrast to the BLA, glucocorticoid-induced memory enhancement for object recognition memory did not require noradrenergic activation within the hippocampus. The hippocampus has been implicated in the detection of novel contexts but not novel objects $(46,47)$, and noradrenergic activity within the hippocampus may be involved in memory of novel contexts (49). Thus, although our findings indicating that object recognition training in a novel environment did not significantly alter pTH and pCREB immunoreactivity levels in the hippocampus may be unexpected, the present findings are consistent with results of lesion studies suggesting that the hippocampus is not, or only minimally, involved in memory of objects $(27,28)$. However, glucocorticoids may interact with noradrenergic mechanisms in the hippocampus in influencing the consolidation of contextual/spatial information. Previous findings have shown that the infusion of either a glucocorticoid receptor agonist or $\beta$-adrenoceptor agonist into the hippocampus enhances memory of such training $(50,51)$. Furthermore, it has been reported that hippocampal excitability (52), as well as the stress-induced modulation of long-term potentiation in the hippocampus, depends on both glucocorticoid and $\beta$-adrenergic influences (53$55)$. However, glucocorticoid effects in the hippocampus on memory consolidation of contextual/spatial training also require concurrent noradrenergic activity within the BLA (12).

The present findings provide increased understanding of the role of glucocorticoids and arousal-induced noradrenergic activation within the BLA in modulating the consolidation of memory for emotionally arousing experiences. As extensive evidence indicates that the memory-modulatory effects of many drugs depend on an intact BLA (5), it seems likely that the present findings may be relevant for other drug systems as well.
These results may thus have important consequences for drug development, because potential cognitive-enhancing drugs may modulate memory consolidation only under conditions that induce sufficient noradrenergic activation of the BLA.

\section{Materials and Methods}

Subjects. Male Sprague-Dawley rats (350-450 g) from Charles River Laboratories were maintained on a 12-h/12-h light/dark cycle (lights on: 0700-1900 h) with ad libitum access to food and water. All procedures complied with National Institutes of Health guidelines and were approved by the University of California, Irvine, Institutional Animal Care and Use Committee.

Object Recognition Test. The experimental apparatus was a gray open-field box $(40 \mathrm{~cm} \times 40 \mathrm{~cm} \times 40 \mathrm{~cm})$ with a sawdust-covered floor, placed in a dimly illuminated room (9). The objects to be discriminated were white glass light bulbs $(6 \mathrm{~cm}$ diameter by 11 $\mathrm{cm}$ length) and transparent glass vials $(5.5 \mathrm{~cm}$ diameter by $5 \mathrm{~cm}$ height). One group of rats was not habituated to the experimental context, whereas the other group was habituated extensively to decrease their novelty stress to the apparatus during the training trial. During habituation, the rats were allowed to freely explore the apparatus in the absence of objects twice per day for 3 min each for 7 days. On the training trial, the rat was placed in the experimental apparatus and allowed to explore two identical objects (A1 and A2) for $3 \mathrm{~min}$. To avoid the presence of olfactory trails, sawdust was stirred and the objects were thoroughly cleaned with $70 \%$ ethanol between rats. Retention was tested after $24 \mathrm{~h}$. One copy of the familiar object (A3) and a new object (B) were placed in the same location as stimuli during the training trial. All combinations and locations of objects were counterbalanced to reduce potential biases because of preference for particular locations or objects. The rat was placed in the experimental apparatus for $3 \mathrm{~min}$ and the time spent exploring each object and the total time spent exploring both objects were recorded. Exploration of an object was defined as pointing the nose to the object at a distance of $<1 \mathrm{~cm}$ and/or touching it with the nose. Turning around, climbing or sitting on an object was not considered as exploration. A discrimination index was calculated as the difference in time exploring the novel and familiar object, expressed as the ratio of the total time spent exploring both objects. Rats showing a total exploration time $<10 \mathrm{~s}$ on either training or testing were excluded.

Cannula Implantation. Rats were implanted bilaterally with guide cannulae aimed at either the BLA [anteroposterior (AP), -2.8 $\mathrm{mm}$ from bregma; mediolateral (ML), $\pm 5.0 \mathrm{~mm}$ from midline; dorsoventral (DV), $-6.5 \mathrm{~mm}$ from skull surface] or dorsal hippocampus [AP, $-3.3 \mathrm{~mm}$; ML, $\pm 1.7 \mathrm{~mm}$; DV,$-2.7 \mathrm{~mm}$ ] based on Paxinos and Watson (56). After behavioral testing, rats were perfused with $4 \%$ formaldehyde, and their brains were sectioned at $50-\mu \mathrm{m}$ thickness, stained with cresyl violet, and examined by light microscopy. Rats $(n=26)$ with injection needle placements outside the BLA or the hippocampus or with extensive tissue damage at the injection needle site were excluded from analyses.

Drug Treatment. Systemically administered drugs were given s.c. (2.0 $\mathrm{ml} / \mathrm{kg})$ immediately after the training trial. Corticosterone $(0.3,1.0$, or $3.0 \mathrm{mg} / \mathrm{kg}$; Sigma) was dissolved in $5 \%$ ethanol in saline, and propranolol (3.0 mg/kg; Sigma) and yohimbine $(0.1,0.3,1.0$, or 3.0 $\mathrm{mg} / \mathrm{kg}$; Sigma) were dissolved in saline. For BLA infusions, the injection needles protruded $2.0 \mathrm{~mm}$ beyond the cannula tip, and a $0.2-\mu \mathrm{l}$ injection volume of saline or propranolol $(0.5 \mu \mathrm{g})$ per hemisphere was infused over $25 \mathrm{~s}$. For hippocampus infusions, the injection needles protruded $1.5 \mathrm{~mm}$ beyond the cannula tip and a $0.5-\mu \mathrm{l}$ injection volume of saline or propranolol $(1.25 \mu \mathrm{g})$ per hemisphere was infused over $35 \mathrm{~s}$. Drug doses were selected on the 
basis of extensive prior evidence (11-13). All drug solutions were freshly prepared before each experiment.

Immunocytochemistry. Rats were perfused with $4 \%$ paraformaldehyde. Selected sections $(25 \mu \mathrm{m})$ were treated with $0.3 \% \mathrm{H}_{2} \mathrm{O}_{2}$, blocked with $3 \%$ normal serum, and the cell membrane was permeabilized with $0.1 \%$ Triton-X100. The primary antibodies TH (rabbit anti-tyrosine hydroxylase, AB151, 1:3000; Chemicon), pTH (rabbit anti-tyrosine hydroxylase phosphoSer19, AB5425, 1:3000; Chemicon; and rabbit anti-tyrosine hydroxylase phosphoSer40, 1:3000, Cell Signaling Technology, Beverly, MA), or pCREB (rabbit anti-Ser-133-phosphorylated CREB, 1:300; Cell Signaling Technology) were applied for $24 \mathrm{~h}$ at $4^{\circ} \mathrm{C}$. Sections were incubated with a biotinylated secondary antibody (goat anti-rabbit or goat anti-mouse, 1:400, Jackson ImmunoResearch) for $2 \mathrm{~h}$ at room temperature, followed by incubation with avidin-biotin complex (1:400, ABC elite kit; Vector Laboratories) for $2 \mathrm{~h}$. Staining was visualized with $20 \mathrm{mg}$ per $100 \mathrm{ml}$ $3,3^{\prime}$-diaminobenzidine (DAB) and $0.03 \% \mathrm{H}_{2} \mathrm{O}_{2}$.

For pTH/dopamine- $\beta$-hydroxylase double labeling, sections were incubated for $24 \mathrm{~h}$ with the primary antibodies pTH and dopamine- $\beta$-hydroxylase (mouse anti-dopamine- $\beta$-hydroxylase, MAB308, 1:1000; Chemicon), followed by Cy5-conjugated donkey anti-rabbit and rhodamine red-conjugated donkey antimouse $\mathrm{F}\left(\mathrm{ab}^{\prime}\right)$ fragments (1:200; Jackson ImmunoResearch). Double labeling was studied by using a Zeiss LSM510 confocal laser and scanning microscope.

1. Heuer, F. \& Reisberg, D. (1990) Mem. Cognit. 18, 496-506.

2. Christianson, S. A., ed. (1992) The Handbook of Emotion and Memory: Research and Theory (Lawrence Erlbaum, Hillsdale, NJ).

3. Roozendaal, B. (2000) Psychoneuroendocrinology 25, 213-238.

4. McGaugh, J. L. \& Roozendaal, B. (2002) Curr. Opin. Neurobiol. 12, 205-210.

5. McGaugh, J. L. (2004) Annu. Rev. Neurosci. 27, 1-28.

6. Buchanan, T. W. \& Lovallo, W. R. (2001) Psychoneuroendocrinology 26, 307-317.

7. Cahill, L., Gorski, L. \& Le, K. (2003) Learn. Mem. 10, 270-274.

8. Rimmele, U., Domes, G., Mathiak, K. \& Hautzinger, M. (2003) NeuroReport 14, 2485-2488.

9. Okuda, S., Roozendaal, B. \& McGaugh, J. L. (2004) Proc. Natl. Acad. Sci. USA 101, 853-858.

10. Abercrombie, H. C., Speck, N. S. \& Monticelli, R. M. (2006) Psychoneuroendocrinology 31, 187-196.

11. Quirarte, G. L., Roozendaal, B. \& McGaugh, J. L. (1997) Proc. Natl. Acad. Sci. USA 94, 14048-14053.

12. Roozendaal, B., Nguyen, B. T., Power, A. E. \& McGaugh, J. L. (1999) Proc. Natl. Acad. Sci. USA 96, 11642-11647.

13. Roozendaal, B., Quirarte, G. L. \& McGaugh, J. L. (2002) Eur. J. Neurosci. 15, 553-560.

14. Cahill, L., Haier, R. J., Fallon, J., Alkire, M. T., Tang, C., Keator, D., Wu, J. \& McGaugh, J. L. (1996) Proc. Natl. Acad. Sci. USA 93, 8016-8021.

15. Hamann, S. B., Ely, T. D., Grafton, S. T. \& Kilts, C. D. (1999) Nat. Neurosci. 2, 289-293.

16. Strange, B. A. \& Dolan, R. J. (2004) Proc. Natl. Acad. Sci. USA 101, 11454-11458.

17. Pelletier, J. G., Likhtik, E., Filali, M. \& Paré, D. (2005) Learn. Mem. 12, 96-102.

18. van Stegeren, A. H., Goekoop, R., Everaerd, W., Scheltens, P., Barkhof, F., Kuijer, J. P. A. \& Rombouts, S. A. R. B. (2005) Neurolmage 24, 898-909.

19. LaBar, K. S. \& Cabeza, R. (2006) Nat. Rev. Neurosci. 7, 54-64.

20. Quirarte, G. L., Galvez, R., Roozendaal, B. \& McGaugh, J. L. (1998) Brain Res. 808, 134-140.

21. McIntyre, C. K., Hatfield, T. \& McGaugh, J. L. (2002) Eur. J. Neurosci. 16, 1223-1226.

22. Ennaceur, A. \& Delacour, J. (1988) Behav. Brain Res. 31, 47-59.

23. Roozendaal, B., Hui, G. K., Hui, I. R., Berlau, D. J., McGaugh, J. L. \& Weinberger, N. M. (2006) Neurobiol. Learn. Mem., in press.

24. Abercrombie, E. D., Keller, R. W., Jr., \& Zigmond, M. J. (1988) Neurosci. 27, 897-904.

25. McEwen, B. S. (2000) Brain Res. 886, 172-189.

26. Lupien, S. J. \& Lepage, M. (2001) Behav. Brain Res. 127, 137-158.

27. Wan, H., Aggleton, J. P. \& Brown, M. W. (1999) J. Neurosci. 19, 1142-1148.

28. Broadbent, N. J., Squire, L. R. \& Clark, R. E. (2004) Proc. Natl. Acad. Sci. USA 101, 14515-14520.
Quantitative Analyses of Immunoreactivity. pTH and TH immunostaining (pTHSer19 immunoreactivity was analyzed because of the better quality of the immunostaining as compared with pTHSer40, although both antibodies showed similar quantitative results) was quantified by using a Quantimet 600 image analysis system, whereas pCREB was quantified by counting the immunopositive nuclei by using an ocular counting frame. Optical density (OD) for pTH and TH and cell counts for pCREB were determined in the BLA and the dentate gyrus and CA1 region of dorsal hippocampus (at bregma level -2.2 to -3.6 , according to standard atlas plates of ref. 56). OD of the immunostained fibers is expressed in arbitrary units corresponding to gray levels. Cell counts are expressed as number of pCREB nuclei per $\mathrm{mm}^{2}$. Quantitative analysis of OD and cell counts were performed blind to the experimental treatment.

Statistics. Data are expressed as mean \pm SEM. Statistical analysis used one- or two-way ANOVAs, followed by post-hoc comparison tests. One-sample $t$ tests assessed whether the discrimination index differed from zero. $P<0.05$ was accepted as statistical significance.

We thank Mary F. Dallman, Richard F. Thompson, and Jerry W. Rudy for helpful comments on a previous draft and Gavin Goei, Jan Gast, and Gabriel Hui for excellent technical assistance. This research was supported by U.S. Public Health Service Grant MH12526 (to J.L.M.), Netherlands Organization for Scientific Research (NWO) Bezoekersbeurs B 95-397 (to B.R.), and NWO Vernieuwingsimpuls 016-021-017 (to E.A.V.d.Z.).

29. Lindgren, N., Xu, Z.-Q. D., Lindskog, M., Herrera-Marschitz, M., Gointy, M., Haycock, J., Goldstein, M., Hökfelt, T. \& Fisone, G. (2000) J. Neurochem. 74 2470-2477.

30. Nagatsu, T., Levitt, M. \& Udenfriend, S. (1964) J. Biol. Chem. 239, 2910-2917.

31. Johnson, L. R., Farb, C., Morrison, J. H., McEwen, B. S. \& LeDoux, J. E. (2005) Neuroscience 136, 289-299.

32. Roseboom, P. H. \& Klein, D. C. (1995) Mol. Pharmacol. 47, 439-449.

33. Davies, M. F., Tsui, J., Flannery, J. A., Li, X., DeLorey, T. M. \& Hoffman, B. B. (2004) Neuropsychopharmacology 29, 229-239.

34. Josselyn, S. A., Shi, C., Carleson, W. A., Jr., Neve, R. L., Nestler, E. J. \& Davis, M. (2001) J. Neurosci. 21, 2404-2412.

35. Saha, S. \& Datta, S. (2005) Eur. J. Neurosci. 21, 3403-3414.

36. Abercrombie, H. C., Kalin, N. H., Thurow, M. W. Rosenkranz, M. A. \& Davidson, R. J. (2003) Behav. Neurosci. 117, 505-516.

37. de Kloet, E. R. (2000) Eur. J. Pharmacol. 405, 187-198.

38. Impey, S., Smith, D. M., Obrietan, K., Donahue, R., Wade, C. \& Storm, D. R. (1998) Nat. Neurosci. 1, 595-601.

39. Huang, Y. Y., Martin, K. C. \& Kandel, E. R. (2000) J. Neurosci. 20, 6317-6325.

40. Barnes, P. J. (1998) Clin. Sci. 94, 557-572.

41. Colangelo, A. M., Mallei, A., Johnson, P. F. \& Mocchetti, I. (2004) Mol. Brain Res. 124, 97-104.

42. Liang, K. C., Chen, L. L. \& Huang, T. E. (1995) Chin. J. Physiol. 38, 81-91.

43. Ferry, B., Roozendaal, B. \& McGaugh, J. L. (1999) J. Neurosci. 19, 5119-5123.

44. LaLumiere, R. T., Buen, T. V. \& McGaugh, J. L. (2003) J. Neurosci. 23, 6754-6758.

45. Miranda, M. I. \& McGaugh, J. L. (2004) Learn. Mem. 11, 312-317.

46. Zhu, X. O., Brown, M. W., McCabe, B. J. \& Aggleton, J. P. (1995) Neuroscience 69, 821-829.

47. Zhu, X. O., McCabe, B. J., Aggleton, J. P. \& Brown, M. W. (1997) Neurosci. Lett. 229, 141-143.

48. Bermudez-Rattoni, F., Okuda, S., Roozendaal, B. \& McGaugh J. L. (2005) Learn. Mem. 12, 447-449.

49. Straube, T., Korz, V., Balschun, D. \& Frey, J. U. (2003) J. Physiol. 552, 953-960.

50. Bevilaqua, L., Ardenghi, P., Schröder, N., Bromberg, E., Schmidz, P. K., Schaeffer, E., Quevedo, J., Bianchin, M., Walz, R., Medina, J. H. \& Izquierdo, I. (1997) Behav. Pharmacol. 8, 331-338.

51. Roozendaal, B. \& McGaugh, J. L. (1997) Eur. J. Neurosci. 9, 76-83.

52. Joëls, M. \& de Kloet, E. R. (1989) Science 245, 1502-1505.

53. Akirav, I. \& Richter-Levin, G. (2002) J. Neurosci. 22, 9912-9921.

54. Korz, V. \& Frey, J. U. (2003) J. Neurosci. 23, 7281-7287.

55. Korz, V. \& Frey, J. U. (2005) J. Neurosci. 25, 7393-7400.

56. Paxinos, G. \& Watson, C. (1997) The Rat Brain in Stereotaxic Coordinates (Academic, San Diego), 3rd Ed. 